\title{
Hyperglycaemia and cancer risk
}

A recent Swedish prospective study on hyperglycaemia and cancer risk (1) showed an association with total cancer risk in women, and in women and men combined, for several cancer sites. The cancer incidence was compared with both fasting glucose and glucose $2 \mathrm{~h}$ after a $75 \mathrm{~g}$ glucose load. For women the relative total cancer risks for the top versus bottom quartiles were in the range of 1.3 , i.e. a $30 \%$ increased risk. In men, however, the most common form, prostate cancer, was inversely related to hyperglycaemia, which made the overall glycaemia/cancer relationship not significant. The study was from the Västerbotten cohort. Almost 70000 men and women were enrolled and followed for 13 years. All were analysed for fasting and postload glucose concentrations. The associations were independent of body mass index, which showed only a very modest correlation with glucose levels.

The study confirms previous reports on associations between diabetes, as well as prediabetic increased plasma glucose levels, and increased risk of several cancers. As concluded by the authors, the results suggest that abnormal glucose metabolism is a general risk factor for cancer development. The fact that plasma glucose levels remained associated with cancer risk after adjustment for body mass index would favour public health strategies aimed at decreasing plasma glucose levels.
Lifestyle changes incorporating moderate weight reduction and increased physical activity have been shown to be effective in reducing type 2 diabetes and may also have an impact on cancer risks. Dietary changes to decrease weight are obviously important, but it is more difficult to make recommendations on dietary changes specifically to decrease fasting glucose and glucose levels $2 \mathrm{~h}$ after a glucose load, i.e. dietary changes that increase insulin sensitivity and/or insulin release. Reduction in sugar or carbohydrate intake would not in itself provide this effect as automatically concluded in media reports. Low-carbohydrate diets would rather decrease the insulin sensitivity. However, a decrease in saturated fat has been shown to improve insulin sensitivity, and a low glycaemic index may be important as well.

Nils-Georg Asp

\section{Reference}

1. Stattin P, Björ O, Ferrari P, Lukanova A, Lenner P, Lindahl B, et al. Prospective study of hyperglycemia and cancer risk. Diabetes Care 2007; 30: 561-7. 